\title{
POINTWISE BOUNDS FOR A SOLUTION OF A NONLINEAR BOUNDARY VALUE PROBLEM IN PARTIAL DIFFERENTIAL EQUATIONS
}

\author{
N. ANDERSON and A. M. ARTHURS'
}

(Received 7 May 1982; revised 24 August 1982)

\begin{abstract}
Pointwise bounds are obtained for the solution of a Dirichlet problem involving the nonlinear Liouville equation in the plane. Illustrative calculations are performed for a square domain.
\end{abstract}

\section{Introduction}

Consider the boundary value problem described by the equations

$$
\begin{gathered}
\nabla^{2} u=e^{u} \text { in } V, \\
u=1 \text { on } B,
\end{gathered}
$$

where $V$ is some closed domain in the plane with $B$ as its boundary. Taken on its own, the differential equation (1.1) possesses an exact solution due to Liouville [3], and for simple domains $V$ this can be used to solve the complete boundary value problem in (1.1) and (1.2). For instance the exact solution of the problem when $V$ is a disc has been obtained by Hart [2]. For other domains it may not be possible to find the exact solution and in such cases approximate solutions must be sought, using numerical or variational approaches for example. The main drawback of these approaches, however, is that extensive computation is often required. Here we shall adopt a very much simpler approach and present analytical pointwise bounds on the exact solution by using the maximum principle for differential equations [1]. Illustrative results are obtained in a simple case.

\footnotetext{
1 Department of Mathematics, University of York, Heslington, York, Y0I 5DD, England.

(c) Copyright Australian Mathematical Society 1983
} 


\section{General pointwise bounds}

The boundary value problem can be written as

$$
-\nabla^{2} u=f(u) \text { in } V \text {, }
$$

with

$$
u=1 \text { on } B
$$

where

$$
f(u)=-e^{u} .
$$

We note that $f(u)$ is a continuous function of $u$, and

$$
\partial f / \partial u=-e^{u}<0 .
$$

At the end of this section we establish the existence and uniqueness of a solution of (2.1) and (2.2). We shall denote the solution by $u$. Now suppose that two functions $u_{1}$ and $u_{2}$ can be found so that

$$
-\nabla^{2} u_{1} \leqslant f\left(u_{1}\right) \text { in } V, \quad u_{1}=1 \text { on } B,
$$

and

$$
-\nabla^{2} u_{2} \geqslant f\left(u_{2}\right) \quad \text { in } V, \quad u_{2}=1 \quad \text { on } B .
$$

Then (2.5) and (2.1) with (2.2) imply

$$
-\nabla^{2}\left(u_{1}-u\right) \leqslant f\left(u_{1}\right)-f(u) \leqslant-K\left(u_{1}-u\right)
$$

for $K \geqslant 0$, since $\partial f / \partial u<0$. Hence

$$
-\nabla^{2}\left(u_{1}-u\right)+K\left(u_{1}-u\right) \leqslant 0,
$$

implying, by the maximum principle, that

$$
u_{1}-u \leqslant 0
$$

or

$$
u_{1} \leqslant u \text {. }
$$

In the same way, with (2.6) instead of (2.5), we find that

$$
u \leqslant u_{2} \text {. }
$$

Combining these we therefore have shown that the upper and lower bounds

$$
u_{1}(x, y) \leqslant u(x, y) \leqslant u_{2}(x, y) \text { in } V
$$

are valid.

One way to obtain bounding functions $u_{1}$ and $u_{2}$ in practice is to choose two functions $f_{1}$ and $f_{2}$ such that

$$
f_{1}(u) \leqslant f(u) \text { for all } u,
$$


and

$$
f_{2}(u) \geqslant f(u) \text { for all } u,
$$

and let $u_{1}$ and $u_{2}$ be solutions of

$$
-\nabla^{2} u_{1}=f_{1}\left(u_{1}\right) \text { in } V
$$

and

$$
-\nabla^{2} \ddot{u}_{2}=f_{2}\left(u_{2}\right) \text { in } V \text {, }
$$

subject to the boundary conditions (2.2). Because of (2.8) and (2.9) the inequalities in (2.5) and (2.6) are then satisfied. The functions $f_{1}$ and $f_{2}$ can be taken to be linear functions, and so (2.10) and (2.11) are readily solved analytically for the bounding functions $u_{1}$ and $u_{2}$. This procedure for obtaining $u_{1}$ and $u_{2}$ has been exploited recently in one-dimensional problems by Villadsen and Michelsen [5] and by Varma and Streider [4].

The existence of a solution to the problem in (2.1) and (2.2) can be established by using a result due to Amman [1]. To apply this result we rewrite (2.1) and (2.2) in the form

$$
-\nabla^{2} v=g(v) \text { in } V, \quad v=0 \text { on } B
$$

where

$$
g(v)=e^{1-v}, \quad v=1-u .
$$

Since $g(0)=e>0$, and the boundary condition satisfies $v \geqslant 0$ trivially, Amman's theorem $\mathrm{A}$ enables us to state that a necessary and sufficient condition for the existence of a non-negative solution $v$ of the boundary value problem (2.12) is the existence of a non-negative $C^{2}$ function $w$ satisfying

$$
-\nabla^{2} w \geqslant g(w) \text { in } V, \quad w \geqslant 0 \text { on } B .
$$

To construct such a function $w$ we consider the problem

$$
-\nabla^{2} w=g_{2}(w)=e \text { in } V, \quad w=0 \quad \text { on } B .
$$

Since $-\nabla^{2} w>0$ here, it follows that $w$, if it exists, cannot have a minimum in $V$, and therefore

$$
w \geqslant 0 \text { in } V \text {. }
$$

For such values of $w$,

$$
g_{2}(w) \geqslant g(w),
$$

and so (2.14) is satisfied. In addition, (2.15) has the explicit solution

$$
w(x, y)=\sum_{\substack{n=1 \\ \text { odd } n}}^{\infty} \frac{16 e}{n^{3} \pi^{3}}(-1)^{(n+1) / 2} \cos \frac{n \pi x}{2}\left(\frac{\cosh n \pi y / 2}{\cosh n \pi / 2}-1\right) .
$$


This function $w$ satisfies (2.16) and (2.14), and hence by Amman's theorem there exists a non-negative solution $v$ of (2.12). Since $v=1-u$, we have therefore established the existence of a solution $u$ to the problem in (2.1) and (2.2).

To show that the solution $u$ of

$$
\nabla^{2} u=e^{u} \quad \text { in } V, \quad u=1 \text { on } B,
$$

is unique we suppose the contrary and let $u_{\alpha}$ and $u_{\beta}$ denote two distinct solutions of the boundary value problem. If we define the quantity

$$
P_{\alpha \beta}=\int_{V}\left[\left\{\operatorname{grad}\left(u_{\alpha}-u_{\beta}\right)\right\}^{2}+\left(u_{\alpha}-u_{\beta}\right)\left(e^{u_{\alpha}}-e^{u_{\beta}}\right)\right] d V,
$$

we see that for any distinct functions $u_{\alpha}$ and $u_{\beta}$

$$
P_{\alpha \beta}>0 \text {. }
$$

But, using the divergence theorem in (2.20), we have

$$
\begin{aligned}
P_{\alpha \beta}= & \int_{V}\left(u_{\alpha}-u_{\beta}\right)\left\{-\nabla^{2}\left(u_{\alpha}-u_{\beta}\right)+e^{u_{\alpha}}-e^{u_{\beta}}\right\} d V \\
& +\int_{B}\left(u_{\alpha}-u_{\beta}\right) \frac{\partial}{\partial n}\left(u_{\alpha}-u_{\beta}\right) d B,
\end{aligned}
$$

and if $u_{\alpha}$ and $u_{\beta}$ are distinct solutions of (2.19) then

$$
P_{\alpha \beta}=0 \text {, }
$$

which contradicts (2.21). Hence the solution $u$ of (2.19) is unique.

\section{Pointwise bounds}

To illustrate the procedure for finding bounding functions we shall consider the problem when $V$ is the region

$$
V=\{-1 \leqslant x \leqslant 1,-1 \leqslant y \leqslant 1\}
$$

in cartesian coordinates, with boundary $B$. Since by (1.1) $\nabla^{2} u>0$, the exact solution $u$ cannot have a maximum in $V$, and it therefore follows that

$$
u \leqslant 1 \text { in } V \text {. }
$$

Figure 1 shows the relevant part of the $u, f(u)$ diagram for $f(u)=-e^{u}$, where the parameter $a<1$ will be determined later. 


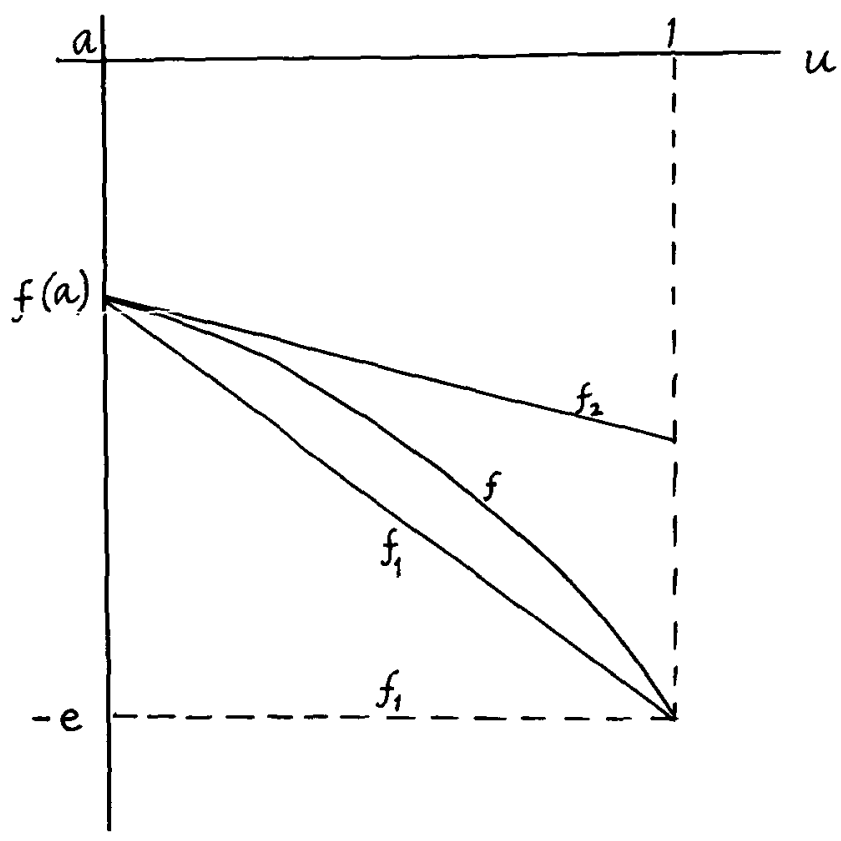

Figure 1. The relevant part of the $u, f(u)$ diagram for $f(u)=e^{-u}$.

\subsection{Lower bounding function}

To obtain a pointwise lower bound $u_{1}$ for $u$ we first choose the function $f_{1}(u)$ as the horizontal line in Figure 1 through the point $(1,-e)$. This line lies below the function $f(u)=-e^{u}$ in the region specified by (3.2) and is given by

$$
f_{1}(u)=-e \text {. }
$$

For this choice of $f_{1}(u)$ the inequality $(2.8)$ holds and by $(2.10)$ the function $u_{1}$ satisfies

$$
\begin{gathered}
\nabla^{2} u_{1}=e \text { in } V \\
u_{1}=1 \text { on } B .
\end{gathered}
$$

Equations (3.4) and (3.5) have the solution

$$
u_{1}(x, y)=1+\sum_{\substack{n=1 \\ \text { odd } n}}^{\infty} \frac{16 e}{n^{3} \pi^{3}}(-1)^{(n-1) / 2} \cos \frac{n \pi x}{2}\left(\frac{\cosh (n \pi y / 2)}{\cosh (n \pi / 2)}-1\right) \text {, }
$$

which from the theory of Section 2 is a lower bounding function for the exact solution $u$ of (1.1) and (1.2). The smallest value of $u_{1}(x, y)$ in (3.6) occurs at $x=0, y=0$, and is given by

$$
\begin{aligned}
u_{1}(0,0) & =0 \cdot 198966 \\
& =a_{0} \quad \text { say }
\end{aligned}
$$


and this therefore provides a global lower bound on $u$. Thus we now know that

$$
a_{0} \leqslant u \leqslant 1 \text { in } V \text {. }
$$

Referring to Figure 1 we see that we can confine $u$ to the region (3.8) by choosing the value of the parameter $a$ appropriately. If we do this we can improve on the bounding function in (3.6) by replacing the function $f_{1}(u)$ in (3.3) by the chord joining the points $(a, f(a))$ and $(1,-e)$. This chord lies below the function $f(u)=-e^{u}$ for the range (3.8) and is given by

$$
f_{l}(u)=-k^{2}(u-a)+f(a),
$$

where

$$
k^{2}=-\frac{f(1)-f(a)}{1-a}=\frac{e-e^{a}}{1-a} .
$$

For this choice of $f_{1}(u)$ the inequality $(2.8)$ holds and by $(2.10)$ the function $u_{1}$ satisfies

$$
\begin{gathered}
\nabla^{2} u_{1}=k^{2}\left(u_{1}-a\right)-f(a) \text { in } V, \\
u_{1}=1 \text { on } B .
\end{gathered}
$$

The solution of (3.11) and (3.12) is

$$
u_{1}(x, y)=a+k^{-2} f(a)+\left(1-a-k^{-2} f(a)\right) w_{1}(x, y),
$$

where

$$
f(u)=-e^{u}, \quad k^{2}=\frac{e-e^{a}}{1-a},
$$

and

$$
w_{1}(x, y)=1+\sum_{\substack{n=1 \\ \text { odd } n}}^{\infty} \frac{4 k^{2}}{n \pi k_{n}^{2}}(-1)^{(n-1) / 2} \cos \frac{n \pi x}{2}\left(\frac{\cosh k_{n} y}{\cosh k_{n}}-1\right),
$$

with

$$
k_{n}^{2}=k^{2}+\frac{n^{2} \pi^{2}}{4} .
$$

With $a=a_{0}$ given by (3.7), we find from (3.13) that the exact function $u$ is bounded below by

$$
\begin{aligned}
u_{1}\left(0,0 ; a=a_{0}\right) & =0.436983 \\
& =a_{1} \text { say. }
\end{aligned}
$$

This allows us to make an immediate improvement in the lower bound function by working in the region

$$
a_{1} \leqslant u \leqslant 1
$$


instead of that in (3.8). With $a=a_{1}$ we find from (3.13) that the exact function $u$ is bounded below by

$$
\begin{aligned}
u_{1}\left(0,0 ; a_{1}\right) & =0 \cdot 455458 \\
& =a_{2} \quad \text { say. }
\end{aligned}
$$

Continuing in this way we generate a sequence $\left\{a_{i}\right\}$ of global lower bounds on $u$ by

$$
a_{\imath+1}=u_{1}\left(0,0 ; a_{i}\right), \quad i=0,1,2, \ldots
$$

The numbers $a_{1}$, form a monotonic increasing sequence that is bounded above. This sequence therefore has a limit which after generating $a_{3}, a_{4}, \ldots, a_{9}$ we find to be

$$
a=0.457094
$$

to six places of decimals. This therefore is the best value of $a_{i}$ to take in the lower bound calculation based on a single chord. Some values of the corresponding lower bounding function are given in Table 1.

TABLE 1. Lower bounding function $u_{1}(x, y)=u_{1}(y, x)$.

\begin{tabular}{c|ccccccc} 
& $\pm x$ & 0.0 & 0.2 & 0.4 & 0.6 & 0.8 & $1 \cdot 0$ \\
\hline $\pm y$ & & & & & & & \\
$0 \cdot 0$ & & 0.457 & 0.473 & 0.524 & 0.618 & 0.769 & $1 \cdot 0$ \\
$0 \cdot 2$ & & & 0.488 & 0.537 & 0.628 & 0.774 & $1 \cdot 0$ \\
$0 \cdot 4$ & & & & 0.580 & 0.660 & 0.792 & $1 \cdot 0$ \\
$0 \cdot 6$ & & & & & 0.721 & 0.826 & $1 \cdot 0$ \\
$0 \cdot 8$ & & & & & & 0.886 & $1 \cdot 0$ \\
1.0 & & & & & & & $1 \cdot 0$ \\
\hline
\end{tabular}

\subsection{Upper bounding function}

To obtain a pointwise upper bound $u_{2}$ for $u$ we choose the function $f_{2}(u)$ as the tangent to $f(u)$ at the point $(a, f(a))$ where $a$ is given by (3.21). From Figure 1 we see that this tangent lies above $f(u)$ and is given by

$$
f_{2}(u)=-K^{2}(u-a)+f(a),
$$

where

$$
K^{2}=-f^{\prime}(a)=e^{a} .
$$

For this choice of $f_{2}(u)$ the inequality (2.9) holds and by (2.11) the function $u_{2}$ satisfies

$$
\begin{gathered}
\nabla^{2} u_{2}=K^{2}\left(u_{2}-a\right)-f(a) \text { in } V, \\
u_{2}=1 \text { on } B .
\end{gathered}
$$

As for (3.11) and (3.12) this boundary value problem has the solution

$$
u_{2}(x, y)=a+K^{-2} f(a)+\left(1-a-K^{-2} f(a)\right) w_{2}(x, y),
$$


where

$$
f(u)=-e^{u}, \quad K^{2}=e^{a},
$$

and

$$
w_{2}(x, y)=1+\sum_{\substack{n=1 \\ \text { odd } n}}^{\infty} \frac{4 K^{2}}{n \pi K_{n}^{2}}(-1)^{(n-1) / 2} \cos \frac{n \pi x}{2}\left(\frac{\cosh K_{n} y}{\cosh K_{n}}-1\right),
$$

with

$$
K_{n}^{2}=K^{2}+\frac{n^{2} \pi^{2}}{4}
$$

Some values of the function $u_{2}$ are given in Table 2, the parameter $a$ being given by (3.21).

TABLE 2. Upper bounding function $u_{2}(x, y)=u_{2}(y, x)$.

\begin{tabular}{l|lllllll}
$\mathrm{c}$ & $\pm x$ & 0.0 & 0.2 & 0.4 & 0.6 & 0.8 & $1 \cdot 0$ \\
\hline $\pm y$ & & & & & & & \\
$0 \cdot 0$ & & 0.470 & 0.486 & 0.537 & 0.631 & 0.779 & $1 \cdot 0$ \\
0.2 & & & 0.502 & 0.551 & 0.641 & 0.784 & $1 \cdot 0$ \\
0.4 & & & & 0.594 & 0.673 & 0.801 & 1.0 \\
0.6 & & & & & 0.733 & 0.835 & $1 \cdot 0$ \\
0.8 & & & & & & 0.893 & $1 \cdot 0$ \\
1.0 & & & & & & & $1 \cdot 0$ \\
\hline
\end{tabular}

From Tables 1 and 2 we see that the exact solution $u$ has been determined to within a few per cent throughout the domain $V$. These are the best bounds that can be obtained by taking a single chord and single tangent in the $u, f(u)$ diagram. To improve on these it is necessary to introduce more elaborate functions $f_{1}$ and $f_{2}$, but, since the main ideas have been illustrated, we shall not pursue this further here.

\section{References}

[1] H. Amman, "On the existence of positive solutions of nonlinear elliptic boundary value problems", Indiana Univ. Math. J. 21 (1971), 125-146.

[2] V. G. Hart, "Exact solutions of two nonlinear equations and hypercircle estimates", J. Austral. Math. Soc. Ser. B 22 (1980), 98-103.

[3] J. Liouville, "Sur l'équation aux différences partielles $\left[\partial^{2} /(\partial u \partial v)\right](\log \lambda) \pm \lambda / 2 a^{2}=0$ ", $J$. de Math. 18 (1853), 71-72.

[4] A. Varma and W. Streider, "Approximate solutions of nonlinear boundary value problems", IMA J. Appl. Math. (in press).

[5] J. Villadsen and M. L. Michelsen, Solution of differential equation models by polynomial approximation (Prentice-Hall, Englewood Cliffs, N.J., 1978). 